
allemande

47-1 | 2015

L'Allemagne et l'Europe | La déprise de l'Empire napoléonien en Allemagne en 1813

\title{
La nouvelle règlementation allemande sur les énergies renouvelables
}

chronique juridique

\section{Sandie Calme}

\section{OpenEdition \\ Journals}

Édition électronique

URL : https://journals.openedition.org/allemagne/485

DOI : 10.4000/allemagne.485

ISSN : 2605-7913

Éditeur

Société d'études allemandes

\section{Édition imprimée}

Date de publication : 26 juin 2015

Pagination : $243-247$

ISSN : 0035-0974

Référence électronique

Sandie Calme, «La nouvelle règlementation allemande sur les énergies renouvelables », Revue

d'Allemagne et des pays de langue allemande [En ligne], 47-1 | 2015, mis en ligne le 13 décembre 2017, consulté le 20 mai 2021. URL : http://journals.openedition.org/allemagne/485 ; DOI : https://doi.org/ 10.4000/allemagne.485 


\section{Chronique juridique}

\section{La nouvelle règlementation allemande sur les énergies renouvelables}

\section{- Sandie Calme*}

Le droit allemand est mu par une volonté de favoriser le développement des énergies renouvelables, de manière propre à soutenir durablement l'activité économique.

D'après l'article 20a de la Loi fondamentale, qui date du 27 octobre 1994, l'État protège les fondements vitaux naturels et en assume la responsabilité envers les générations futures. Cet article de la Loi fondamentale est considéré comme un moteur de l'action des pouvoirs législatif, exécutif et judiciaire en faveur des énergies renouvelables ${ }^{(1)}$.

La promotion des énergies renouvelables aspire à rendre l'utilisation de l'électricité aussi écologique que possible, tout en limitant la dépendance envers des combustibles fossiles qui se font de plus en plus rares, en préservant sa fiabilité et en la maintenant abordable. Ce sont les objectifs poursuivis par la loi sur les énergies renouvelables (Erneuerbare-Energien-Gesetz, EEG) entrée en vigueur en 2010.

Cette loi de 2010 a favorisé l'implantation des énergies renouvelables en Allemagne de manière significative. Sa réforme au $1^{\mathrm{er}}$ août $2014^{(2)}$ vise à en maîtriser les coûts, à en planifier le développement et à favoriser l'accès de ces énergies au marché. Partant du constat que le prix de l'électricité est un facteur de compétitivité pour les entreprises énergivores et, en considération de la concurrence internationale, il est porté une attention particulière à ce que la création de valeurs ainsi que les emplois soient pérennisés en Allemagne. L'accent est mis sur les énergies éolienne et photovoltaïque.

* Docteur en droit, LL.M. (Francfort-sur-le-Main, Allemagne), avocate au barreau de Paris.

1 Voir Klaus Hansmann, Dieter Sellner (dir.), Grundzüge des Umweltrechts, Berlin, 2012 (4éd.), $\mathrm{n}^{\circ} 46$, p. 26.

2 Bundesministerium für Wirtschaft und Energie, EEG-Reform : Planbar. Bezahlbar. Effizient. 


\section{Les objectifs de pérennisation des énergies renouvelables}

Les énergies renouvelables sont mises à contribution du développement durable.

Jusqu'en 2025, la part d'énergie renouvelable doit se situer entre 40 et $45 \%$, jusqu'en 2035 entre 55 et $60 \%$.

Les objectifs sont les suivants :

- une croissance annuelle de 2,5 gigawatts bruts pour l'énergie solaire,

- une croissance annuelle de 2,5 gigawatts nets pour l'énergie éolienne terrestre,

- une croissance annuelle d'environ 100 mégawatts pour la bioénergie,

- une installation d'énergie éolienne en mer de 6,5 gigawatts jusqu'en 2020 et de 15 gigawatts jusqu'en 2030 .

\section{L'agenda en dix points}

La réforme est basée sur un agenda en dix points (Zentrale Vorhaben Energiewende für die 18. Legislaturperiode, 10-Punkte-Energie-Agenda des Bundesministeriums für Wirtschaft und Energie) présenté par le ministère fédéral de l'Économie pour la dixhuitième législature.

Les dix points sont les suivants:

\section{1. les énergies renouvelables}

Afin que les techniques propres aux énergies renouvelables fassent l'objet, principalement, d'appels d'offres concurrentiels, un projet pilote est lancé qui doit aboutir à une modification de la loi sur les énergies renouvelables sur la base de ses résultats, fin 2016. Ce procédé d'appels d'offres concurrentiels doit être transparent et faire l'objet d'une large participation des acteurs concernés.

\section{2. le cadre européen pour le climat et l'énergie à l'horizon 2030}

La réforme de la loi sur les énergies renouvelables s'inscrit dans le cadre des évolutions de l'Union européenne, avec notamment une forte demande de concertation sur un objectif de réduction des émissions de gaz à effet de serre d'au moins $40 \%$ jusqu'en 2030 ainsi qu'un seuil d'exploitation des énergies renouvelables porté à $30 \%$ à cette même date.

\section{3. la réforme de la bourse européenne du carbone}

La présente réforme prend en considération et soutient la réforme de la bourse européenne du carbone.

\section{4. la configuration du marché de l'électricité}

Les centrales électriques doivent à l'avenir préserver leur efficacité et présenter des gages de sécurité face à la croissance des énergies renouvelables, ce qui doit faire l'objet d'une consultation publique, ainsi que d'échanges avec les États voisins et avec la Commission européenne, en vue d'une réforme législative.

\section{5. la stratégie d'efficacité}

L'efficacité énergétique doit être renforcée, et ce en particulier sous l'égide du plan d'action national pour l'efficacité énergétique (Nationaler Aktionsplan Energieeffizienz, NAPE), qui fixe les objectifs et les responsabilités des différents acteurs, renforce le 
fonds dédié à l'efficacité énergétique comme instrument de financement et définit les outils de financement adaptés. À cet égard, il est tenu compte de la transposition des dispositions de la directive 2012/27/UE du Parlement européen et du Conseil du 25 octobre 2012 relative à l'efficacité énergétique.

\section{6. la stratégie immobilière}

L'accent est porté sur la mise aux normes des immeubles en matière énergétique, que ce soit en ce qui concerne l'électricité, le chauffage ou l'optimisation de la consommation énergétique, ce qui passe par le redéploiement de la loi sur le chauffage aux énergies renouvelables (Erneuerbare-Energien-Wärmegesetz, EnEV) et de l'ordonnance sur les économies d'énergie (Energieeinsparverordnung, EEWärmeG).

\section{7. les réseaux de transfert}

Planifier le développement du réseau énergétique pour les années à venir, c'est mâ̂triser la célérité de la construction d'équipements spécifiques, leur répartition géographique entre les Länder et le développement du parc conventionnel de centrales électriques.

Cela passe par la concertation auprès de l'Agence du réseau fédéral (Bundesnetzagentur, BNetzA) qui élabore un plan de développement du réseau (Netzentwicklungsplan, NEP).

À cette fin, la loi fédérale de planification de la demande (Bundesbedarfsplangesetz) doit être modifiée en 2016.

\section{8. les réseaux de distribution}

Pour ce qui est de l'agencement des réseaux de distribution des énergies renouvelables, une ordonnance sur la régulation de l'attractivité (Anreizregulierungsverordnung, ARegV) est envisagée pour 2015, sur la base du rapport d'évaluation de l'Agence du réseau fédéral et des résultats de l'étude portant sur la modernisation des réseaux de distribution pour l'Allemagne (Moderne Verteilernetze für Deutschland).

\section{9. le monitoring}

Le processus de monitoring Energie der Zukunft (énergie du futur) mis en place par le gouvernement fédéral vise à observer dans la continuité et en détail l'évolution énergétique pour pouvoir intervenir tout au long de cette évolution, au moyen notamment de rapports annuels évaluant les progrès par rapport aux objectifs dans leur ensemble ainsi que la transposition des mesures requises.

\section{0. les plateformes du tournant énergétique}

Les plateformes du tournant énergétique sont mises en place afin de permettre aux groupes sociaux de prendre part au développement politique. Il s'agit de forums et de plateformes de discussion du ministère fédéral de l'Économie et de l'Environnement. Il est envisagé d'organiser au total cinq plateformes du tournant énergétique, sur les thèmes de l'efficacité, des réseaux énergétiques, des immeubles, de la recherche et de l'innovation. 


\section{La maîtrise des coûts}

Les coûts de la mutation énergétique doivent être supportés à la fois par le client privé et par l'industrie.

Pour les entreprises énergivores, une règlementation d'exception est mise en place appelée Besondere Ausgleichsregelung ${ }^{(3)}$ qui leur permet de limiter voire de réduire à néant leur contribution fiscale au titre de la loi sur les énergies renouvelables, en adéquation avec le droit de l'Union européenne. À l'avenir, ce privilège d'exception sera cantonné aux entreprises énergivores sujettes à la concurrence internationale, afin de préserver la compétitivité et les emplois. Ainsi, les entreprises favorisées de la sorte verseront pleinement la contribution pour la première gigawatt heure et en principe $15 \%$ de celle-ci pour l'électricité qu'elles auront consommée au-delà, mais tout au plus $4 \%$ de la création de valeur brute de l'entreprise concernée ou tout au plus $0,5 \%$ pour ce qui est des entreprises assumant des frais de consommation électrique d'au moins $20 \%$.

L'ordonnance sur les taxes a ainsi dû être modifiée. Elle inclut également les chemins de fer.

L'énergie photovoltaïque, l'énergie éolienne terrestre et la bioénergie bénéficient d'une marge appelée «atmender Deckel »: s'il y a plus d'équipements d'énergie renouvelable que prévu, les montants de cotisations pour les équipements futurs diminueront automatiquement. Par contre, les seuils de cotisation sont fixes pour l'énergie éolienne en mer.

Les entreprises sont autorisées à solliciter une réduction de leur cotisation au titre de la loi sur les énergies renouvelables: pour 2015, les données de 2013 servent de bases de calcul, en considération de la contribution en vigueur en 2013.

L'obligation pour les exploitants d'équipements nouveaux d'une certaine taille de commercialiser directement l'énergie qu'ils produisent porte sur les équipements suivants :

- depuis le $1^{\text {er }}$ août 2014, tous les équipements nouveaux à partir de prestations de 500 kilowatts,

- dès le $1^{\text {er }}$ janvier 2016, tous les équipements nouveaux à partir de prestations de 100 kilowatts.

\section{Des règlementations de compensation spécifiques aux chemins de fer}

Pour ce qui est des chemins de fer, une loi spéciale est envisagée (Entwurf eines Ersten Gesetzes zur Änderung des Erneuerbare-Energien-Gesetzes) pour qu'à l'avenir, les chemins de fer nouveaux entrants sur le marché allemand puissent bénéficier des privilèges de cette règlementation compensatoire. La Commission européenne a validé ce projet sous réserve d'un changement de la loi sur les énergies renouvelables ouvrant la possibilité de requérir le bénéfice de la règlementation compensatoire par avance, par exemple concernant les entreprises désirant prendre part à un appel d'offres public pour des prestations de services de circulation ferroviaire.

3 Bundesministerium für Wirtschaft und Energie, Informationen zur Besonderen Ausgleichsregelung, 15 octobre 2014 ; Martin Geipel, Maximilian Uibeleisen, « Praxisrelevante Neuerungen der besonderen Ausgleichsregelung nach dem EEG 2014 für Stromkosten und handelsintensive Unternehmen », NJW, 2014, p. 3276 sq. 


\section{La production d'électricité pour le compte du producteur}

Les nouveaux équipements de fourniture d'électricité pour le propre compte du fournisseur sont amenés à participer financièrement à l'aménagement des énergies renouvelables.

En matière de production d'électricité pour le propre compte du producteur, en faisant usage d'équipements propres aux énergies renouvelables ou pour les équipements de pointe procédant par la cogénération de chaleur et d'électricité, la contribution est réduite dans son montant, à savoir $30 \%$ en 2015 et $35 \%$ en 2016, étant entendu que tous les équipements de cogénération de chaleur et d'électricité de pointe comme les équipements utilisant les énergies renouvelables sont assujettis à une contribution de $40 \%$ à partir de 2017.

\section{Le point de vue de l'Union européenne}

Le projet de loi sur les énergies renouvelables a fait l'objet d'échanges, afin de garantir sa conformité avec le droit européen des aides d'État, avec la Commission européenne (Bruxelles, 25.11.2014, C(2014) 8822 final). Celle-ci a établi que les entreprises énergivores ne devaient payer a posteriori que pour une fraction des avantages qui leur sont garantis en 2013 et 2014. Ce faisant, la Commission a exigé des remboursements partiels en en fixant le mode de calcul.

Les appels d'offres sont conçus comme ouverts aux projets venant de l'étranger, notamment en provenance d'États membres de l'Union européenne, qui sont concernés par le régime des cotisations énergétiques. 